Urologe 2021 · 60:901-909

https://doi.org/10.1007/s00120-021-01479-8

Angenommen: 18. Januar 2021

Online publiziert: 2. März 2021

(C) Der/die Autor(en) 2021, korrigierte

Publikation 2021

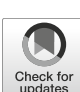

Matthias Jahnen ${ }^{1}$ Lorenz Dichtl ${ }^{1}$. Nora Stirenberg ${ }^{1}$ Andreas Dinkel ${ }^{2}$.

Stefan Schiele' · Helga Schulwitz ${ }^{1}$ - Jürgen E. Gschwend' · Kathleen Herkommer ${ }^{1}$

'Klinik und Poliklinik für Urologie, Klinikum rechts der Isar, Fakultät für Medizin, Technische Universität München, München, Deutschland

${ }^{2}$ Klinik und Poliklinik für Psychosomatische Medizin und Psychotherapie, Klinikum rechts der Isar, Fakultät für Medizin, Technische Universität München, München, Deutschland

\title{
Selbstuntersuchung von Hoden und Brust - eine retrospektive Kohortenstudie an Medizinstudierenden
}

selber regelmäßig abtasten oder durch einen Arzt abgetastet werden [10, 19, 25]. Es konnte außerdem gezeigt werden, dass Männer mit geringer Bildung und niedrigem sozioökonomischen Status seltener eine Selbstuntersuchung der Hoden durchführen und dass durch die öffentliche Sensibilisierung für das Thema Hodenkrebs die Bereitschaft junger Erwachsener zur Selbstuntersuchung in den vergangenen Jahrzehnten gestiegen ist $[10,19]$. Dennoch sind Jungen und junge Männer hinsichtlich Hodenkrebs häufig unzureichend informiert und in den meisten Fällen auch nicht an einen Urologen angebunden, so dass eine individuelle Beratung bzgl. Vorsorge, Früherkennung und urologischer Gesundheit oftmals unterbleibt [19].

Im Vergleich dazu ist aufgrund von Vorsorgeuntersuchungen im Rahmen der Zervixkarzinomprävention und der Verschreibungspflicht hormoneller Kontrazeptiva die frauenärztliche Anbindung junger Frauen häufiger gegeben [5]. Diese regelmäßigen Arztbesuche ermöglichen es jungen Frauen außerdem, frühzeitig hinsichtlich Brustkrebs und regelmäßiger Selbstuntersuchung der Brust beraten zu werden. Zahlen zum Selbstuntersuchungsverhalten junger Frauen in Deutschland ebenso wie eine allgemeingültige Empfehlung zur Selbstuntersuchung der Brust als Früherkennungsmaßnahme fehlen aber [6, 15].
Es ist wichtig, junge Erwachsene zu motivieren, sich eigenständig $\mathrm{zu}$ untersuchen und für das Thema Hodenbzw. Brustkrebs zu sensibilisieren, um die Rate an frühdiagnostizierten, kurativ behandelbaren Tumoren $\mathrm{zu}$ steigern. Diesbezüglich scheint die öffentliche Informationsverbreitung mittels Aufklärungskampagnen ein wichtiger Schritt. Inwiefern darüber hinaus aber andere Faktoren, insbesondere sexuelle Aufgeschlossenheit, Sexualkundeunterricht und das Sexualleben, die Bereitschaft für eine regelmäßige Selbstuntersuchung beeinflussen können, ist zum Großenteil noch unklar. In dieser Analyse wurde das Selbstuntersuchungsverhalten einer medizinisch gebildeten Kohorte ermittelt und Faktoren, welche im Zusammenhang mit regelmäßiger Selbstuntersuchung der Hoden oder Brust stehen, identifiziert.

\section{Studiendesign und Unter- suchungsmethoden}

\section{Design und Prozedere}

Bei der vorliegenden Studie handelt es sich um eine Querschnittsuntersuchung mit einer anfallenden Stichprobe. Im Rahmen der medizinischen Lehre der Klinik und Poliklinik für Urologie des Klinikums rechts der Isar wurden Medizinstudierende der Technischen Universität München des 4. und 5. StuMänner im Alter von 18-35 Jahren sich 
dienjahrs während des Praktikumstags (April 2018 bis Februar 2020) mittels anonymisierter Fragebögen bezüglich ihres Gesundheits- und Sexualverhaltens befragt. Die Fragebögen für männliche und weibliche Studierende waren bis auf einzelne geschlechtsspezifische Fragen gleich. In diese Analyse wurden alle Studierenden eingeschlossen, die die Frage zur Selbstuntersuchung beantwortet hatten. Die Ethikkommissen der Technischen Universität München hat der vorliegenden Studie zugestimmt.

\section{Instrumente}

Zur Erfassung des Selbstuntersuchungsverhaltens wurden die männlichen Studierenden gefragt, ob sie regelmäßig ihre Hoden untersuchen bzw. die weiblichen Studierenden gefragt, ob sie regelmäßig ihre Brust untersuchen (nein; ja).

Die folgenden soziodemografischen Faktoren und Lebensstilfaktoren wurden in die Analyse eingeschlossen: Alter (Jahre), Wohnsituation (bei den Eltern; Wohngemeinschaft; mit PartnerIn/ Familie; Wohnheim; eigene Wohnung), Partnerschaft (nein; ja; ja, Fernbeziehung), Kinder (nein; ja), BMI $\left(\mathrm{kg} / \mathrm{m}^{2}\right)$, Zufriedenheit mit dem eigenen Aussehen (Skala von 1 [sehr unzufrieden] bis 10 [sehr zufrieden]).

Des Weiteren wurden die Studierenden gefragt, ob sie mit ihrer/ihrem PartnerIn, ihren Eltern, ihren FreundInnen oder mit Fremden über die eigene sexuelle Aktivität kommunizieren (nein; ja). Die Teilnahme an einem Sexualkundeunterricht zur Schulzeit wurde erhoben (nein; ja). Außerdem wurde erfasst, ob die Studierenden die Notwendigkeit für mehr Aufklärungsunterricht in der Schule sehen (nein; ja).

Hinsichtlich sexueller Orientierung und des Sexualverhaltens wurden folgende Parameter in die Analyse eingeschlossen: sexuelle Orientierungsidentität (heterosexuell; eher heterosexuell; bisexuell; eher homosexuell; homosexuell), sexuell bevorzugtes Geschlecht (weiblich; männlich; anderes), vaginaler Geschlechtsverkehr (jemals im Leben; [nein; ja]), vaginaler Geschlechtsverkehr (in den letzten 4 Wochen; nein; ja), Masturbationshäufigkeit in den letzten
4 Wochen, Verwendung von Kondomen (im letzten Jahr; nein; ja), Gebrauch einer Notfallkontrazeption (im letzten Jahr; nein; ja), Nachweis einer sexuell übertragbaren Erkrankung (jemals im Leben; nein; ja). Die männlichen Studierenden wurden außerdem gefragt, ob sie unter einer eigeschränkten Erektionsfähigkeit (Penis bei der Erektion nicht komplett hart), an einem frühzeitigen Samenerguss (innerhalb einer 1 min nach vaginaler Penetration) oder an Schmerzen während des Geschlechtsverkehrs leiden (jeweils: nein; ja). Das Beantworten einer oder mehrerer dieser Fragen mit ,ja“" wurde als mögliche Beeinträchtigung der sexuellen Funktion definiert.

\section{Statistische Auswertung}

Zur Beschreibung der Stichprobe erfolgte die Darstellung kategorialer Parameter in absoluten Zahlen und Prozent. Stetige Parameter wurden als Mittelwert plus Standardabweichung bzw. Median plus Interquartilsbereich dargestellt. Assoziationen zwischen den erhobenen Parametern und dem Selbstuntersuchungsverhalten männlicher sowie weiblicher Studierender wurde mittels logistischer Regressionsanalyse berechnet. $P$-Werte $\leq 0,05$ wurden als signifikant definiert. Die statistische Auswertung erfolgte mittels SAS (Version 9.4, SAS Institute Inc, Cary, NC, USA).

\section{Ergebnisse}

Die Fragebögen wurden von $98,9 \%$ aller $(n=482)$ Medizinstudierenden, die am Praktikumstag teilnahmen, ausgefüllt; 4 Studierende wurden aufgrund eines unzureichend ausgefüllten Fragebogens ausgeschlossen. Insgesamt wurden 473 durchschnittlich 25 Jahre alte (MW $=24,9$ Jahre, $\mathrm{SD}=2,9$ Jahre) Medizinstudierende in diese Analyse eingeschlossen (177 männliche und 296 weibliche Studierende; 0 Tab. 1).

Es gaben $64,2 \%$ der männlichen Studierenden an, regelmäßig ihre Hoden zu untersuchen und $72,2 \%$ der weiblichen Studierenden gaben an, regelmäßig ihre Brust abzutasten (• Abb. 1).
Mehr als 90,0\% der männlichen sowie weiblichen Studierenden gaben an, mit ihrem/ihrer PartnerIn über die eigene Sexualität zu sprechen bzw. zu sprechen, wenn sie eine/einen hätten. Kommunikation über Sexualität mit FreundInnen wurde von mehr als drei Viertel der Studierenden angegeben (männliche Studierende: $76,1 \%$ vs. weibliche Studierende: $85,7 \%)$. Jeder fünfte der Studierenden gab an, mit seinen/ihren Eltern über die eigene Sexualität zu sprechen und nur eine Minderheit der Studierenden gab an, mit Fremden über die eigene Sexualität zu sprechen (12,6\%). Zwar gaben mehr als zwei Drittel der männlichen und weiblichen Studierenden an, an Sexualkundeunterricht in der Schule teilgenommen zu haben, aber die meisten Studierenden sahen trotzdem die Notwendigkeit für mehr Aufklärung in der Schule (•Tab. 1).

Die meisten Studierenden gaben an, eine heterosexuelle bzw. eine eher heterosexuelle Orientierungsidentität zu haben. Die meisten Studierenden waren sexuell erfahren und gaben an, schon einmal vaginalen Geschlechtsverkehr im Leben $(91,4 \%)$ bzw. in den letzten 4 Wochen gehabt zu haben (73,3\%). Bei 15,3\% der männlichen Studierenden zeigte sich der Hinweis auf eine beeinträchtigte sexuelle Funktion (• Tab. 1).

In der univariaten Analyse zeigten sich einzelne, für weibliche und männliche Studierende spezifische Assoziationen mit einer regelmäßigen Selbstuntersuchung der Hoden bzw. Brust. Männliche Studierende, die angaben, nicht mit ihrer/ ihrem PartnerIn über die eigene Sexualität zu sprechen, führten seltener eine Selbstuntersuchung durch $(p<0,05)$. Eine ähnliche Assoziation zeigte sich bei weiblichen Studierenden, die eine fehlende sexuelle Kommunikation mit FreundInnen angaben $(p<0,05$; $\bullet$ Tab. 2 und $\bullet$ Abb. 2).

Außerdem führten männliche Studierende, die bei ihren Eltern wohnten und jene, die keinen vaginalen Geschlechtsverkehr in den letzten 4 Wochen hatten, signifikant seltener eine regelmäßige Selbstuntersuchung der Hoden durch $(p<0,05)$. Auch zeigte sich der Trend, dass männliche Studierende mit dem Hinweis auf eine eingeschränkte sexu- 
Urologe 2021·60:901-909 https://doi.org/10.1007/s00120-021-01479-8

(c) Der/die Autor(en) 2021

M. Jahnen · L. Dichtl · N. Stirenberg · A. Dinkel · S. Schiele · H. Schulwitz · J. E. Gschwend · K. Herkommer

\section{Selbstuntersuchung von Hoden und Brust - eine retrospektive Kohortenstudie an Medizinstudierenden}

\section{Zusammenfassung}

Hintergrund. Durch eine regelmäßige Selbstuntersuchung kann ein Malignom der Hoden oder Brust gegebenenfalls frühzeitig erkannt und kurativ behandelt werden. Ziel dieser Arbeit war es, das Selbstuntersuchungsverhalten von Medizinstudierenden zu untersuchen und Faktoren, die eine regelmäßige Selbstuntersuchung beeinflussen, zu identifizieren.

Methodik. Medizinstudierende wurden mittels Fragebogen bezüglich ihres Gesundheitsund Sexualverhaltens befragt. 98,8\% der Studierenden nahmen teil $(n=473)$. Die erhobenen Daten wurden mittels univariater und multivariater logistischer Regression analysiert.
Ergebnisse. Es gaben $64,2 \%$ der männlichen Studierenden $(n=177)$ an, regelmäßig ihre Hoden zu untersuchen und $72,2 \%$ der weiblichen Studierenden $(n=296)$ gaben an, regelmäßig ihre Brust abzutasten. Studierende, die nicht mit ihrer/ihrem Partnerln bzw. mit ihren Freundlnnen über ihre Sexualität sprechen, führten seltener eine Selbstuntersuchung durch $(p<0,05)$. Männliche Studierende, die in den letzten 4 Wochen vor Befragung keinen Geschlechtsverkehr hatten und weibliche Studierende, die im gleichen Zeitraum nicht masturbierten, führten seltener eine Selbstuntersuchung durch $(p<0,05)$.

Diskussion. Der Anteil an Medizinstudierenden, der eine regelmäßige
Selbstuntersuchung durchführt, ist im Vergleich zu weniger medizinisch gebildeten jungen Erwachsenen hoch. Wissen über die Relevanz von Hoden- bzw. Brustkrebs scheint grundlegend für eine regelmäßige Selbstuntersuchung zu sein. Ein belastetes Sexualleben schränkt solch ein Gesundheitsverhalten möglicherweise ein. Eine Verbesserung der Aufklärung über Hodenkrebs und die urologische Anbindung von Jungen/junger Männer bieten daher die Möglichkeit, die Bereitschaft zur Selbstuntersuchung innerhalb dieser Altersgruppe zu steigern.

Schlüsselwörter

Hodenkrebs · Brustkrebs · Selbstuntersuchung $\cdot$ Früherkennung $\cdot$ Sprechstunde

\section{Testicular and breast self-examination-a retrospective cohort study of medical students}

\section{Abstract}

Background. Regular self-examination can facilitate early detection of testicular cancer and malignancies of the breast and may ensure a curative treatment. In this analysis we explored the tendencies of medical students to perform self-examination and associated factors.

Methods. As part of their urology rotation, medical students of the Technical University of Munich were surveyed via questionnaires regarding their health and sexual behavior. In all, $98.8 \%$ of the students participated and data from 473 of 477 students were included in this analysis. Data were analyzed using univariate and multivariate regression analysis.
Results. In all, $64.2 \%(n=177)$ of the male students and $72.3 \%(n=296)$ of the female students performed regular self-examination of the testis and breast, respectively. Students who did not communicate with their partners or friends about their sex lives were less likely to preform regular self-examination $(p<0.05)$. Male students without sexual intercourse in the 4 weeks prior to the survey and female students who did not masturbate in the 4 weeks prior to the survey were also less likely to preform regular self-examination $(p<0.05)$. Discussion. The rate of regular self-examination is high in medical students compared to previous studies on young adults and nonmedical students. This shows that knowledge about the significance of testicular cancer and breast cancer are fundamental for promoting self-examination in teenagers and young adults. A distressed sex life might hinder young adults in preforming regular selfexamination. Therefore, improved education about the significance of testicular cancer and routine urological consultations for male teenagers and young men are ways to promote testicular self-examination within this age group.

\section{Keywords}

Testicular cancer - Self-examination - Early detection - Breast cancer $\cdot$ Health awareness elle Funktion seltener eine regelmäßige Selbstuntersuchung der Hoden durchführten ( $p=0,06$; $\bullet$ Tab. 2 und $\bullet$ Abb. 2).

Bei weiblichen Studierenden zeigte sich eine signifikante Assoziation zwischen einer Partnerschaft bzw. dem Zusammenleben mit PartnerIn/Familie und dem Unterlassen einer regelmäßigen Selbstuntersuchung der Brust $(p<0,05$; - Tab. 2 und - Abb. 2).

Es zeigten sich bei Studierenden beider Geschlechter keine signifikanten Assoziationen zwischen der Teilnahme an einem Sexualkundeunterricht und einer regelmäßigen Selbstuntersuchung der Hoden bzw. Brust (• Tab. 2).

In der multivariaten Regression zeigte sich bei männlichen Studierenden eine signifikante Assoziation zwischen fehlender Kommunikation mit dem/der PartnerIn und fehlender Selbstuntersuchung der Hoden (OR: 0,11 [0,02-0,95]). Bei weiblichen Studieren zeigte sich in der multivariaten Analyse, dass insbesondere weibliche Studierende, die in den letzten 4 Wochen nicht mastur- biert hatten (OR: 0,43 [0,24-0,78]) und jene, die das weibliche als sexuell bevorzugtes Geschlecht angaben (OR: 0,26 $[0,07-0,96])$, seltener eine Selbstuntersuchung der Brust durchführen (alles $p<0,05$; Abb. 2).

\section{Diskussion}

Eine regelmäßige Selbstuntersuchung der Hoden ermöglicht es jungen Männern, eine Veränderung der Hoden frühzeitig zu erkennen, sodass ein mögliches 
Tab. 1 Charakteristika des Studienkollektivs

\begin{tabular}{|c|c|c|c|c|c|c|}
\hline & \multicolumn{2}{|c|}{$\begin{array}{l}\text { Männliche Studierende } \\
(n=177)\end{array}$} & \multicolumn{2}{|c|}{$\begin{array}{l}\text { Weibliche Studierende } \\
(n=296)\end{array}$} & \multicolumn{2}{|c|}{$\begin{array}{l}\text { Gesamtkohorte } \\
(n=473)\end{array}$} \\
\hline & $n$ & $\%$ & $n$ & $\%$ & $n$ & $\%$ \\
\hline \multicolumn{7}{|l|}{ Regelmäßige Selbstuntersuchung (Hoden/Brust) } \\
\hline Nein & 63 & 35,8 & 82 & 27,8 & 145 & 30,8 \\
\hline $\mathrm{Ja}$ & 113 & 64,2 & 213 & 72,2 & 326 & 69,2 \\
\hline \multicolumn{7}{|l|}{ Soziodemographie und Lebensstilfaktoren } \\
\hline Alter (Jahre) MW (SD) & \multicolumn{2}{|l|}{$25,1(3,2)$} & \multicolumn{2}{|l|}{$24,8(2,8)$} & \multicolumn{2}{|l|}{$24,9(2,9)$} \\
\hline $\mathrm{BMI}\left(\mathrm{kg} / \mathrm{m}^{2}\right) \mathrm{MW}(\mathrm{SD})$ & \multicolumn{2}{|l|}{$23,4(2,7)$} & \multicolumn{2}{|l|}{$21,0(2,8)$} & \multicolumn{2}{|l|}{$21,9(3,0)$} \\
\hline $\begin{array}{l}\text { Zufriedenheit mit dem eigenen Aussehen (1-10) } \\
\text { MW (SD) }\end{array}$ & \multicolumn{2}{|l|}{$7,3(1,5)$} & \multicolumn{2}{|l|}{$7,0(1,7)$} & \multicolumn{2}{|l|}{$7,1(1,6)$} \\
\hline \multicolumn{7}{|l|}{ Wohnsituation } \\
\hline Bei den Eltern & 9 & 5,1 & 26 & 8,9 & 35 & 7,5 \\
\hline Wohngemeinschaft & 74 & 42,3 & 121 & 41,3 & 195 & 41,7 \\
\hline Mit PartnerIn/Familie & 31 & 17,7 & 55 & 18,8 & 86 & 18,4 \\
\hline Wohnheim & 14 & 8,0 & 20 & 6,8 & 34 & 7,3 \\
\hline Eigene Wohnung & 47 & 26,9 & 71 & 24,2 & 118 & 25,2 \\
\hline \multicolumn{7}{|l|}{ Partnerschaft } \\
\hline Nein & 63 & 36,0 & 92 & 31,2 & 155 & 33,0 \\
\hline $\mathrm{Ja}$ & 99 & 56,6 & 185 & 62,7 & 284 & 60,4 \\
\hline Fernbeziehung & 13 & 7,4 & 18 & 6,1 & 31 & 6,6 \\
\hline \multicolumn{7}{|l|}{ Kinder } \\
\hline Nein & 165 & 95,9 & 287 & 98,3 & 452 & 97,4 \\
\hline $\mathrm{Ja}$ & 7 & 4,1 & 5 & 1,7 & 12 & 2,6 \\
\hline \multicolumn{7}{|l|}{ Sexuelle Kommunikation und Bildung } \\
\hline \multicolumn{7}{|l|}{ Kommunikation über Sexualität mit PartnerIn } \\
\hline Nein/Nein (kein/e Partnerln) & 7 & 4,0 & 17 & 5,8 & 24 & 5,1 \\
\hline Ja/Ja (kein/e Partnerln) & 168 & 96,0 & 277 & 94,2 & 445 & 94,9 \\
\hline \multicolumn{7}{|l|}{ Kommunikation über Sexualität mit den Eltern } \\
\hline Nein & 144 & 82,3 & 234 & 79,6 & 378 & 80,6 \\
\hline Ja & 31 & 17,7 & 60 & 20,4 & 91 & 19,4 \\
\hline \multicolumn{7}{|l|}{ Kommunikation über Sexualität mit FreundInnen } \\
\hline Nein & 42 & 23,9 & 42 & 14,3 & 84 & 17,9 \\
\hline $\mathrm{Ja}$ & 134 & 76,1 & 252 & 85,7 & 386 & 82,1 \\
\hline \multicolumn{7}{|l|}{ Kommunikation über Sexualität mit Fremden } \\
\hline Nein & 147 & 84,0 & 263 & 89,5 & 410 & 87,4 \\
\hline Ja & 28 & 16,0 & 31 & 10,5 & 59 & 12,6 \\
\hline Sexualkundeunterricht in der Schule oder dem S & ium & & & & & \\
\hline Nein & 48 & 27,3 & 78 & 26,9 & 126 & 27,0 \\
\hline $\mathrm{Ja}$ & 128 & 72,7 & 212 & 73,1 & 340 & 73,0 \\
\hline Notwendigkeit für mehr Aufklärung in der Schul & & & & & & \\
\hline Nein & 47 & 27,0 & 41 & 14,1 & 88 & 19,0 \\
\hline Ja & 127 & 73,0 & 249 & 85,9 & 376 & 81,0 \\
\hline Sexualleben und sexuelle Gesundheit & & & & & & \\
\hline Sexuelle Orientierungsidentität & & & & & & \\
\hline Heterosexuell & 145 & 82,9 & 227 & 77,5 & 372 & 79,5 \\
\hline Eher heterosexuell & 18 & 10,3 & 55 & 18,8 & 73 & 15,6 \\
\hline Bisexuell & 3 & 1,7 & 8 & 2,7 & 11 & 2,4 \\
\hline Eher homosexuell/homosexuell & 9 & 5,1 & 3 & 1,0 & 12 & 2,6 \\
\hline
\end{tabular}




\begin{tabular}{|c|c|c|c|c|c|c|}
\hline & \multicolumn{2}{|c|}{$\begin{array}{l}\text { Männliche Studierende } \\
(n=177)\end{array}$} & \multicolumn{2}{|c|}{$\begin{array}{l}\text { Weibliche Studierende } \\
(n=296)\end{array}$} & \multicolumn{2}{|c|}{$\begin{array}{l}\text { Gesamtkohorte } \\
(n=473)\end{array}$} \\
\hline & $n$ & $\%$ & $n$ & $\%$ & $n$ & $\%$ \\
\hline Weiblich & 158 & 90,8 & 12 & 4,2 & 170 & 36,8 \\
\hline Männlich & 15 & 8,6 & 276 & 95,8 & 291 & 63,0 \\
\hline Anderes & 1 & 0,6 & 0 & 0,0 & 1 & 0,2 \\
\hline \multicolumn{7}{|l|}{ Vaginaler Geschlechtsverkehr (jemals im Leben) } \\
\hline Nein & 15 & 8,6 & 25 & 8,6 & 40 & 8,6 \\
\hline $\mathrm{Ja}$ & 160 & 91,4 & 267 & 91,4 & 427 & 91,4 \\
\hline \multicolumn{7}{|l|}{ Vaginaler Geschlechtsverkehr (in den letzten 4 Wochen) } \\
\hline Nein & 49 & 28,3 & 74 & 25,7 & 123 & 26,7 \\
\hline $\mathrm{Ja}$ & 124 & 71,7 & 214 & 74,3 & 338 & 73,3 \\
\hline $\begin{array}{l}\text { Masturbationshäufigkeit (in den letzten } 4 \text { Wochen; } \\
\text { [n] M [IQR]) }\end{array}$ & $10(6-20)$ & & $3(1-5)$ & & $5(2-8)$ & \\
\hline \multicolumn{7}{|l|}{ Verwendung von Kondomen (im letzten Jahr) } \\
\hline Nein & 54 & 30,7 & 116 & 39,3 & 170 & 36,1 \\
\hline $\mathrm{Ja}$ & 122 & 69,3 & 179 & 60,7 & 301 & 63,9 \\
\hline \multicolumn{7}{|l|}{ Notfallkontrazeption (im letzten Jahr) } \\
\hline Nein & 162 & 92,1 & 263 & 89,2 & 425 & 90,2 \\
\hline $\mathrm{Ja}$ & 14 & 7,9 & 32 & 10,8 & 46 & 9,8 \\
\hline \multicolumn{7}{|l|}{ Sexuell übertragbare Erkrankung (jemals im Leben) } \\
\hline Nein & 145 & 82,9 & 250 & 85,0 & 395 & 84,2 \\
\hline Ja & 30 & 17,1 & 44 & 15,0 & 74 & 15,8 \\
\hline \multicolumn{7}{|l|}{ Beeinträchtigte sexuelle Funktion } \\
\hline Nein & 149 & 84,7 & - & & - & \\
\hline $\mathrm{Ja}$ & 27 & 15,3 & - & & - & \\
\hline
\end{tabular}

Malignom in einem frühen Stadium diagnostiziert und behandelt werden kann $[11,14]$. Der regelmäßigen Durchführung solch einer Selbstuntersuchung scheinen insbesondere Wissen über Hodenkrebs und ein hohes Bildungsniveau zugrunde zu liegen $[10,19,24]$. In unserer Studie gaben $64,2 \%$ der befragten männlichen Medizinstudierenden an, regelmäßig ihre Hoden zu untersuchen. Dieser Anteil liegt damit deutlich höher als in vorangegangenen Studien. In Studien aus den USA und Irland gaben $36,0 \%$ der Männer im Alter von 18-35 Jahren bzw. 18-67 Jahren an mehrmals im Jahr eine Selbstuntersuchung der Hoden durchzuführen $[8,10$, 25]. In einer Studie an deutschen Studierenden gaben $48,9 \%$ der männlichen Teilnehmer an, solch eine Untersuchung schon einmal durchgeführt zu haben. Im Vergleich mit diesen Studien ist es wichtig zu beachten, dass in unsere Analyse ausschließlich Daten von Medizinstudierenden des 4. bis 5. Studienjahrs, die bereits an der Urologie-Vorlesung teilgenommen hatten, eingeflossen sind. In diesem Kollektiv kann man daher davon ausgehen, dass das Wissen über Hodenkrebs, Vorsorge und das Verständnis für Gesundheitsverhalten höher ist als in der Allgemeinbevölkerung und auch im Vergleich zu Studierenden anderer Fächer $[8,23]$. Dementsprechend unterstreichen die Ergebnisse dieser Analyse, dass ein hohes Bildungsniveau an sich und insbesondere spezifisches medizinisches Wissen die Bereitschaft zur regelmäßigen Selbstuntersuchung bestärken kann.

Ausreichendes Wissen über Hodenkrebs und dessen Relevanz ist bei vielen Jugendlichen und jungen Erwachsenen ohne medizinischen Hintergrund aber nicht gegeben, sodass diesen die Grundlage für das Verständnis für die Durchführung einer regelmäßige Selbstuntersuchung der Hoden fehlt $[1,16$, 17]. Die Entwicklungen der letzten Jahrzehnte deuten aber darauf hin, dass durch öffentliche Wissensvermittlung die Kenntnis der Allgemeinbevölkerung über Hodenkrebs angestiegen ist und auch das Selbstuntersuchungsverhalten gesteigert werden kann [10, 20]. Zusammengenommen zeigt dies, dass es sinnvoll ist, weitere Bemühungen mit der Absicht, Jungen und junge Männer bezüglich Hodenkrebs zu informieren und zur Durchführung einer regelmäBigen Selbstuntersuchung der Hoden zu motivieren. Zum Aufbau eines angemessenen Wissensstands über Hodenkrebs können unterschiedliche Institutionen als mögliche Informationsquelle dienen. Vorangegangene Arbeiten konnten bereits zeigen, dass durch die Informationsverbreitung mit Hilfe von Aufklärungskampagnen das Wissen über Hodenkrebs und die Bereitschaft zur Selbstuntersuchung der Hoden gesteigert werden kann, aber die Reichweite dieser Kampagnen häufig beschränkt ist [10, 19]. Die adäquate Anbindung solcher Kampagnen an soziale Netzwerke wäre ein nächster wichtiger Schritt, um 


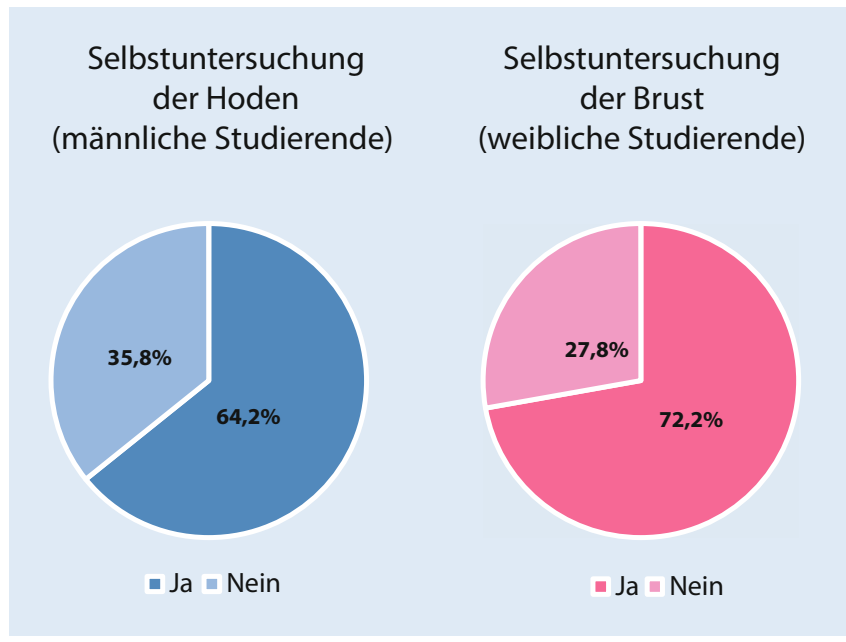

Abb. $1<$ Anteil an männlichen und weiblichen Studierenden, die regelmäßig eine Selbstuntersuchung der Hoden/der Brust durchführen bzw. nicht durchführen
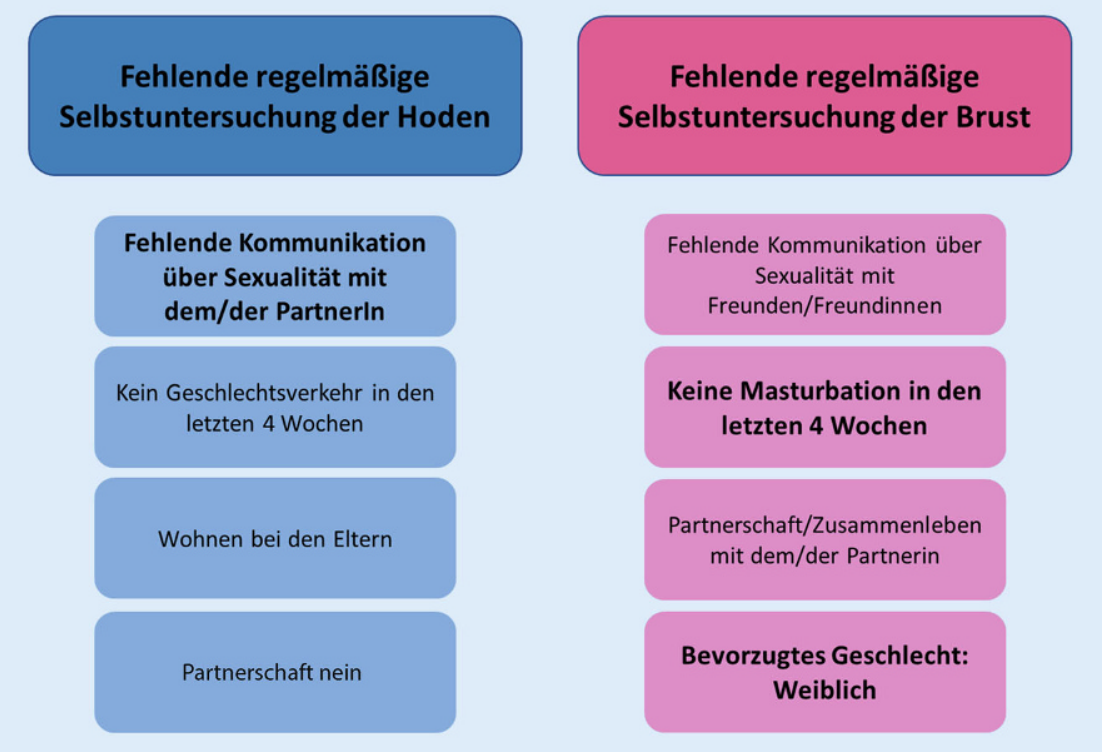

Abb. 2 ム Faktoren, die mit fehlender regelmäßiger Selbstuntersuchung bei männlichen bzw. weiblichen Studierenden assoziiert waren (hervorgehoben sind Faktoren, die in der multivariaten Regression statistisch signifikant waren)

die Zielgruppe der Jungen und jungen Männer besser zu erreichen.

Als eine weitere Informationsquelle für Jungen und junge Männer können ÄrztInnen dienen. Die zweite Jugenduntersuchung (J2) durch den/die Kinderarzt/Kinderärztin im Alter von 16-18 Jahren bietet die Möglichkeit, Gesundheitsvorsorge i. Allg. anzusprechen. Diese Jugenduntersuchung wird aber nur von einem Fünftel der Jugendlichen wahrgenommen und legt den Fokus meist nur unzureichend auf urologische Themen [22]. Der direkte Kontakt zu einem/einer Urologen/Urologin wird von den meisten jungen Männern nur bei akuten Problemen gesucht. Im Gegensatz dazu ist die Anbindung von jungen Frauen an einen/eine Gynäkologen/ Gynäkologin deutlich häufiger gegeben, so dass Fragen zu Themen wie Verhütung, sexuell übertragbare Erkrankungen und Krankheiten des Unterleibs sowie der Brust beantwortet werden können $[9,18]$. Dies trägt möglicherweise dazu bei, dass im Vergleich zu gleichaltrigen Männern Frauen der eigenen Gesundheit häufig auch das Bewusstsein bzgl. der Relevanz von Brutkrebs in der Gesellschaft weiter mehr Aufmerksamkeit schenken und verbreitet ist als Wissen über Hodenkrebs [12]. Zusammengenommen könnte dies dazu führen, dass bereits junge Frauen, obwohl sie nur ein sehr geringes Risiko haben an Brustkrebs zu erkranken, eine hohe Bereitschaft haben, die eigene Brust zu untersuchen. Dies spiegelt sich auch in den Ergebnissen dieser Analyse wider, die zeigen, dass mehr weibliche Studierende $(72,2 \%)$ eine regelmäßige Selbstuntersuchung der Brust durchführen als männliche Studierende $(64,2 \%)$ eine Selbstuntersuchung der Hoden. Um eine ärztliche Versorgung junger Männer ähnlich der von jungen Frauen zu gewährleisten, ist es Ziel der DGU und des Berufsverbandes der Deutschen Urologen (BvDU), eine ,Jungen-Sprechstunde" für Jungen und junge Männer $\mathrm{zu}$ etablieren. In solch einem Rahmen könnte ergänzend zur unpopulären J2Untersuchung eine Beratung hinsichtlich urologischer Gesundheit und eine Anleitung zur Selbstuntersuchung erfolgen und so das Gesundheitsverhalten junger Männer gefördert werden [3]. In Deutschland gibt es bisher eine private Krankenkasse, die einen Sondervertrag zur Hodenkrebsfrüherkennung mit der BvDU abgeschlossen hat [2]. Solche Verträge sollten in Zukunft weiter unterstützt werden, um eine urologische Anbindung junger Männer zu verbessern.

Trotz vergleichbaren Wissensstands gab jeder dritte männliche Studierende an, keine regelmäßige Selbstuntersuchung der Hoden durchzuführen und jede vierte weibliche Studierende gab an, keine regelmäßige Selbstuntersuchung der Brust durchzuführen. Dies deutet darauf hin, dass es weitere Faktoren neben dem Kenntnisstand über Hodenbzw. Brustkrebs gibt, die das Selbstuntersuchungsverhalten beeinflussen können. Solche Faktoren bieten möglicherweise weitere Ansatzpunkte, junge Erwachsene $\mathrm{zu}$ einem bewussteren Gesundheitsverhalten und dem Durchführen einer regelmäßigen Selbstuntersuchung $\mathrm{zu}$ motivieren. In vorangegangenen Studien wurde bereits in einem Kollektiv von Männern im Alter von 18-35 Jahren festgestellt, dass mit steigendem Alter die Bereitschaft zur Selbstuntersuchung der Hoden zunimmt $[20,25]$. Solch eine Assoziation mit dem Alter konnten 
Tab. 2 Mit regelmäßiger Selbstuntersuchung assoziierte Faktoren (univariate Regression)

\section{Charakteristika}

Soziodemographie und Lebensstilfaktoren

Alter (stetig)

BMI (stetig)

Zufriedenheit mit dem eigenen Aussehen (stetig)

Partnerschaft (Ref. nein)

Ja

Fernbeziehung

Kinder (Ref.ja)

Nein

Wohnsituation (Ref. eigene Wohnung)

Bei den Eltern

Wohngemeinschaft

Mit PartnerIn/Familie

Wohnheim

Sexuelle Kommunikation und Bildung

Männliche Studierende

Kommunikation über Sexualität mit PartnerIn (Ref. ja/ja; kein/e Partnerln)

Nein/Nein (kein/e Partnerln)

Kommunikation über Sexualität mit den Eltern (Ref. ja)

Nein

OR (95\%-KI)

$0,96(0,87-1,06)$

$0,98(0,88-1,10)$

$1,06(0,86-1,30)$

$2,06(1,07-3,98)$

$1,37(0,40-4,63)$

$0,70(0,13-3,72) \quad 0,68$

$0,13(0,03-0,72)$

$0,92(0,42-2,00)$

$1,35(0,49-3,71)$

$0,35(0,10-1,20)$

$0,08(0,01-0,73)$

$0,56(0,24-1,35)$

Kommunikation über Sexualität mit FreundInnen (Ref. ja)

Nein

Kommunikation über Sexualität mit Fremden (Ref. ja)

Nein

$0,67(0,33-1,37)$

$0,67(0,27-1,62)$

$1,16(0,58-2,37)$

Nein

Notwendigkeit für mehr Aufklärung in der Schule (Ref. ja)

Nein

$0,54(0,27-1,07)$

Sexualleben und sexuelle Gesundheit

Sexuelle Orientierungsidentität (Ref. heterosexuell)

Eher heterosexuell

Bisexuell

Eher homosexuell/homosexuell

Sexuell bevorzugtes Geschlecht (Ref. weiblich)

Männlich

Sexuell bevorzugtes Geschlecht (Ref. männlich)

Weiblich

Vaginaler Geschlechtsverkehr (jemals im Leben; Ref. ja)

Nein

$0,40(0,15-1,07)$

$0,99(0,09-11,2) \quad 0,99$

$0,62(0,16-2,41) \quad 0,49$

$0,59(0,20-1,72)$

$-$

$0,46(0,16-1,33)$

$0,48(0,24-0,94)$

Nein

$0,99(0,97-1,03)$

Masturbation (in den letzten 4 Wochen; Ref. ja)

Nein

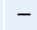

Verwendung von Kondomen (im letzten Jahr)

(Ref.ja)

Nein

$1,17(0,60-2,29)$ p

0,44

0,73

0,58

0,03

0,93

0,02

0,83

0,56

0,09

0,02

0,20

0,28

0,37

0,67

0,08

0,07

0,34

0,15

0,03

0,92

Weibliche Studierende

OR (95\%-KI)

$1,00(0,91-1,10) \quad 0,99$

$1,00(0,91-1,09) \quad 0,94$

$0,98(0,85-1,14) \quad 0,79$

$0,47(0,26-0,86) \quad 0,01$

$4,14(0,52-33,1) \quad 0,08$

$0,65(0,07-5,88) \quad 0,70$

$1,35(0,40-4,55) \quad 0,63$

$0,60(0,30-1,22) \quad 0,16$

$0,43(0,19-0,96) \quad 0,04$

$0,37(0,13-1,07) \quad 0,07$

$0,40(0,15-1,08) \quad 0,07$

$0,68(0,34-1,33) \quad 0,26$

$0,44(0,23-0,87) \quad 0,02$

$1,07(0,47-2,42) \quad 0,88$

$0,79(0,45-1,40) \quad 0,41$

$1,97(0,84-4,66) \quad 0,12$

$1,61(0,78-3,30) \quad 0,20$

$0,67(0,16-2,88) \quad 0,59$

$0,80(0,07-9,00) \quad 0,86$

$0,35(0,11-1,13) \quad 0,08$

$0,80(0,33-1,93) \quad 0,62$

$1,27(0,69-2,33) \quad 0,44$

$-$

$0,45(0,26-0,79) \quad 0,006$

0,65

$1,02(0,60-1,72) \quad 0,94$ 


\section{Originalien}

\begin{tabular}{|c|c|c|c|c|}
\hline \multirow[b]{2}{*}{ Charakteristika } & \multicolumn{2}{|c|}{ Männliche Studierende } & \multicolumn{2}{|c|}{ Weibliche Studierende } \\
\hline & OR (95\%-KI) & $\mathbf{p}$ & OR (95\%-KI) & $p$ \\
\hline \multicolumn{5}{|c|}{ Notfallkontrazeption (im letzten Jahr; Ref. nein) } \\
\hline Ja & $1,43(0,43-4,77)$ & 0,56 & $0,71(0,32-1,54)$ & 0,38 \\
\hline \multicolumn{5}{|c|}{ Sexuell übertragbare Erkrankung (jemals im Leben; Ref. nein) } \\
\hline Ja & $0,97(0,43-2,19)$ & 0,93 & $0,62(0,31-1,21)$ & 0,16 \\
\hline \multicolumn{5}{|c|}{ Beeinträchtigte sexuelle Funktion (Ref. nein) } \\
\hline Ja & $0,46(0,20-1,04)$ & 0,06 & - & - \\
\hline
\end{tabular}

wir aufgrund der schmalen Altersverteilung (MW: 24,9 $\pm 2,9$ Jahre) in unserer Studie nicht darstellen. Es konnte jedoch gezeigt werden, dass männliche Studierende, die noch bei ihren Eltern wohnen, weniger häufig eine Selbstuntersuchung durchführen. Diese Ergebnisse deuten darauf hin, dass eine regelmäßige Selbstuntersuchung nicht unbedingt ein gewisses Alter, sondern evtl. auch ein gewisses $\mathrm{Ma}$ an Selbstständigkeit erfordert. Daher ist es wichtig, bereits Jungen die Verantwortung über ihre eigene Gesundheit bewusst zu machen, um Gesundheitsverhalten wie eine regelmäßige Selbstuntersuchung der Hoden zu fördern.

Des Weiteren sind in dieser Analyse insbesondere Faktoren, die das Sexualverhalten und die sexuelle Aufgeschlossenheit der Studierenden widerspiegeln, berücksichtigt worden. So konnte gezeigt werden, dass männliche Studierende, die nicht mit ihrem/ihrer PartnerIn über die eigene Sexualität sprechen, seltener eine Selbstuntersuchung der Hoden durchführen. Auch weibliche Studierende, die nicht mit ihren FreundInnen über ihre Sexualität sprechen, gaben an, seltener eine Selbstuntersuchung der Brust durchzuführen. Das Verzichten auf solch eine Kommunikation mit dem/der PartnerIn oder FreundInnen kann auf eine gewisse Unsicherheit in Bezug auf die eigene Sexualität hinweisen. Dies geht einher mit einer britischen Studie, die gezeigt hat, dass junge Frauen mit höherer selbstwahrgenommener sexueller Attraktivität häufiger eine Selbstuntersuchung durchführen [7]. Solch eine $\mathrm{Zu}$ rückhaltung im Umgang mit der eigenen Sexualität scheint sowohl jungen Männern als auch junge Frauen in der Bereitschaft zur Selbstuntersuchung, die den aktiven Umgang mit dem eigenen Körper und insbesondere intimer Zonen erfordert, einzuschränken. Dass das Sexualleben möglicherweise Einfluss auf das Selbstuntersuchungsverhalten junger Erwachsener haben kann, zeigt sich auch daran, dass männliche Studierende, die angaben, in den letzten 4 Wochen keinen Geschlechtsverkehr gehabt zu haben und weibliche Studierende, die angaben, in den letzten 4 Wochen nicht masturbiert zu haben, seltener eine Selbstuntersuchung durchführten. Außerdem war in der univariaten Analyse der Trend zu erkennen, dass männliche Studierende, bei denen sich Hinweise auf eine eingeschränkte sexuelle Funktion zeigten, weniger häufig eine regelmäßige Selbstuntersuchung durchführten. Dies deutet darauf hin, dass ein möglicherweise unerfülltes ggf. sogar belastetes Sexualleben einen negativen Einfluss auf das Selbstuntersuchungsverhalten junger Erwachsener haben kann. In diesem Kontext erscheint es umso wichtiger, junge Männer dazu zu motivieren bei sexuellen Problemen den Kontakt zum/zur Urologen/Urologin zu suchen, um die sexuelle Gesundheit zu fördern und so auch positiv auf das allgemeine urologische $\mathrm{Ge}$ sundheitsverhalten einzuwirken. Durch die Etablierung der bereits oben erwähnten urologischen "Jungen-Sprechstunde“ könnte solch eine Selbstverständlichkeit, sich bei sexuellen Problemen an einen/ eine Urologen/Urologin zu wenden, gefördert werden und das Gesundheitsbewusstsein auf unterschiedlichen Ebenen nachhaltig positiv beeinflusst werden.

Zusammenfassend deuten die Ergebnisse dieser Analyse daraufhin, dass es notwendig ist, Jugendliche und junge Erwachsene umfangreich bezüglich sexueller Gesundheit und Hodenkrebs bzw. Brustkrebs aufzuklären sowie sie zu gesundheitsbezogener Selbstständigkeit zu ermutigen, um Gesundheitsverhalten wie das regelmäßige Abtasten von Hoden und Brust zu fördern. Dies sollte bereits in einem gewissen Ausmaß in der Schule und später durch unterschiedliche Bezugspersonen vermittelt werden. Besonders der Kontakt junger Männer mit einem Urologen, über die Versorgung akuter Probleme hinaus sollte z. B. durch eine "Jungen-Sprechstunde“ gefördert werden, um Themen wie Hodenkrebs, Früherkennung und urologische Gesundheitsvorsorge ins Bewusstsein dieser Altersgruppe zu rufen.

Bei Betrachtung der Ergebnisse dieser Arbeit müssen unterschiedliche $\mathrm{Ge}$ sichtspunkte in Betracht gezogen werden. Zum einen handelt sich bei dem Kollektiv um Medizinstudierende, sodass sich die Ergebnisse nicht zwangsläufig auf junge Erwachsene mit anderem Bildungshintergrund übertragen lassen. Des Weiteren wurde die sexuelle Funktion männlicher Studierender nur mit einzelnen Fragen erhoben und ist nicht mit einer klinischen Diagnose gleich zusetzten. Dies ist außerdem nach unserem Kenntnisstand die erste Studie, die den Zusammenhang zwischen dem Sexualverhalten und dem Selbstuntersuchungsverhalten junger Erwachsener untersucht hat, so dass Vergleichsdaten fehlen. Aufgrund der hohen Beteiligungsrate sind die erhobenen Daten aber als sehr valide in Bezug auf deutsche Medizinstudierende einzuschätzen.

\section{Fazit für die Praxis}

\section{- Ein Großteil von Medizinstudieren- den führen eine regelmäßige der}


Selbstuntersuchung der Hoden bzw. Brust durch.

- Ein belastetes Sexualleben kann die Bereitschaft junger Männer zur Selbstuntersuchung der Hoden einschränken.

- Wissen über die Relevanz von Hodenkrebs sollte in der Schule, durch adäquate Hodenkrebskampagnen und Ärzte vermittelt werden.

- Die urologische Anbindung von Jungen und jungen Männern sollte gefördert werden, um diese hinsichtlich der Früherkennung von Hodentumoren und möglicher sexueller Probleme zu beraten.

\section{Korrespondenzadresse}

Prof. Dr. Kathleen Herkommer

Klinik und Poliklinik für Urologie, Klinikum rechts der Isar, Fakultät für Medizin, Technische Universität München

Ismaninger Str. 22, 81675 München,

Deutschland

kathleen.herkommer@tum.de

Funding. Open Access funding enabled and organized by Projekt DEAL.

\section{Einhaltung ethischer Richtlinien}

Interessenkonflikt. M. Jahnen, L. Dichtl, N. Stirenberg, A. Dinkel, S. Schiele, H. Schulwitz, J.E. Gschwend und K. Herkommer geben an, dass kein Interessenkonflikt besteht.

Alle beschriebenen Untersuchungen am Menschen oder an menschlichem Gewebe wurden mit Zustimmung der zuständigen Ethikkommission der TU München durchgeführt. Von allen beteiligten Probanden liegt eine Einverständniserklärung vor.

Open Access. Dieser Artikel wird unter der Creative Commons Namensnennung 4.0 International Lizenz veröffentlicht, welche die Nutzung, Vervielfältigung, Bearbeitung, Verbreitung und Wiedergabe in jeglichem Medium und Format erlaubt, sofern Sie den/die ursprünglichen Autor(en) und die Quelle ordnungsgemäß nennen, einen Link zur Creative Commons Lizenz beifügen und angeben, ob Änderungen vorgenommen wurden.

Die in diesem Artikel enthaltenen Bilder und sonstiges Drittmaterial unterliegen ebenfalls der genannten Creative Commons Lizenz, sofern sich aus der Abbildungslegende nichts anderes ergibt. Sofern das betreffende Material nicht unter der genannten Creative Commons Lizenz steht und die betreffende Handlung nicht nach gesetzlichen Vorschriften erlaubt ist, ist für die oben aufgeführten Weiterverwendungen des $\mathrm{Ma}$ terials die Einwilligung des jeweiligen Rechteinhabers einzuholen.
Weitere Details zur Lizenz entnehmen Sie bitte der Lizenzinformation auf http://creativecommons.org/ licenses/by/4.0/deed.de.

\section{Literatur}

1. Anheuser $P$, Mühlstädt $S$, Fornara $P$ et al (2019) Knowledge and early detection of testicular germ cell cancer among adolescents and young adults. Urologe A 58:1331-1337

2. Betriebskrankenkasse Mobil Oil (2020) Screening der äußeren Genitalien. https://www.bkkmobil-oil.de/leistungen-und-vorteile/vorsorgeund-impfungen/vorsorgeuntersuchungen-fuermaenner\#anchor-id-068b. Zugegriffen: 9. Dez. 2020

3. Deutsche Gesellschaft für Urologie (2019) Jungensprechstunde. https://www.urologenportal. de/jungensprechstunde.html. Zugegriffen: 9. Dez. 2020

4. Leitlinienprogramm Onkologie (Deutsche Krebsgesellschaft, Deutsche Krebshilfe, AWMF) (2020) S3-Leitlinie Diagnostik, Therapie und Nachsorge der Keimzelltumoren des Hodens, Langversion 1.1, AWMF-Registernummer: 043/0490L. https://www.leitlinienprogramm-onkologie.de/ leitlinien/hodentumoren. Zugegriffen: 9. Dez. 2020

5. Leitlinienprogramm Onkologie (Deutsche Krebsgesellschaft, Deutsche Krebshilfe, AWMF) (2020) Prävention des Zervixkarzinoms, Langversion 1.1, AWMF Registernummer: 015/0270L. http://www.leitlinienprogramm-onkologie.de/ leitlinien/zervixkarzinom-praevention/.Zugegriffen:9.Dez. 2020

6. Leitlinienprogramm Onkologie (Deutsche Krebsgesellschaft, Deutsche Krebshilfe, AWMF) (2020) S3-Leitlinie Früherkennung, Diagnose, Therapie und Nachsorge des Mammakarzinoms, Version 4.2, AWMF Registernummer: 032-0450L. http://www.leitlinienprogramm-onkologie.de/ leitlinien/mammakarzinom/. Zugegriffen: 9. Dez. 2020

7. Brewer G, Dewhurst AM (2013) Body esteem and self-examination in British men and women. Int $J$ Prev Med 4:684-689

8. Brown-Kramer CR, Kiviniemi MT (2015) Affective associations and cognitive beliefs relate to individuals' decisions to perform testicular or breast self-exams. J Behav Med 38:664-672

9. Bühmann W (2018) Jungensprechstunde Begleitung für Jungen bei Problemen in der Pubertät. Urologe 57:1221-1221

10. Casey RG, Grainger R, Butler MR et al (2010) Public awareness of testis cancer and the prevalence of testicular self-examination-changing patterns over 20 years. Urology 76:915-918

11. Dieckmann KP (2007) Diagnostic delay in testicular cancer: an analytic chimaera or a worthy goal? Eur Urol 52:1566-1568

12. Ellert U, Wirz J, Ziese T (2006) Beiträge zur Gesundheitsberichterstattung des Bundes - Telefonischer Gesundheitssurvey des Robert Koch-Instituts (2. Welle). https://www. rki.de/DE/Content/Gesundheitsmonitoring/ Gesundheitsberichterstattung/GBEDownloadsB/ gstel04.pdf?_blob=publicationFile. Zugegriffen 9.Dez. 2020

13. Deutsche Gesellschaft für Urologie (2019) Selbstuntersuchung der Hoden: So geht's. https:// www.hodencheck.de/selbstuntersuchung.html. Zugegriffen: 5. Jan. 2021
14. Fadich A, Giorgianni SJ, Rovito MJ et al (2018) USPSTF testicular examination nomination-selfexaminations and examinations in a clinical setting. Am J Mens Health 12:1510-1516

15. Fallenberg M (2020) Diagnostik und Therapie früher und fortgeschrittener Mammakarzinome. https://www.ago-online.de/fileadmin/ ago-online/downloads/_leitlinien/kommission mamma/2020/Alle_aktuellen_Empfehlungen_ 2020.pdf.Zugegriffen: 9.Dez. 2020

16. Grundl S, Kranz J, Rosellen J et al (2018) Wissenserhebung zum Thema Jungengesundheit - zwischen Geschlechterkluft und Nachholbedarf. Urologe 57:1222-1229

17. Kramer H, Lehmann J, Klapp C et al (2018) Gibt's auch 'nen Frauenarzt für Männer? Urologe 57:1230-1239

18. Krause L, Seeling S, Prutz F et al (2017) Prevalence and trends in the utilization of gynecological services by adolescent girls in Germany. Results of the German health survey for children and adolescents (KiGGS). Geburtshilfe Frauenheilkd 77:1002-1011

19. Mani J, Kloft J, Jones J et al (2019) Awareness of clinical relevance of malignant testicular cancer among universitystudents: the value of prevention campaigns. Urologe A 58:790-794

20. McCullagh J, Lewis G, Warlow C (2005) Promoting awareness and practice of testicular selfexamination. Nurs Stand 19:41-49

21. Neal RD, Tharmanathan P, France B et al (2015) Is increased time to diagnosis and treatment in symptomatic cancer associated with poorer outcomes? Systematic review. Br J Cancer 112:S92-S107

22. Kamtsiuris P, Bergmann E, Rattay P, Schlaud M (2007) Inanspruchnahme medizinischer Leistungen: Ergebnisse des Kinder- und Jugendgesundheitssurveys (KiGGS). https://edoc.rki.de/handle/ 176904/432.Zugegriffen: 9. Dez. 2020

23. Vallo S, Kloft J, Jones J et al (2020) Evaluation of testicular self examination and testicular partner examination in medical versus non-medical students. Curr Urol 14:92-97

24. Ward KD, Vander Weg MW, Read MC et al (2005) Testicular cancer awareness and self-examination among adolescent males in a community-based youth organization. Prev Med 41:386-398

25. Wynd CA (2002) Testicular self-examination in young adultmen. JNurs Scholarsh 34:251-255 